\title{
Synchronous Cholecystectomy and Totally Extraperitoneal (TEP) Herniorrhaphy Using an Umbilical Incision
}

\author{
Hae-Hyeon Suh, M.D., Ph.D. ${ }^{1}$, Yong Kwon Cho, M.D. ${ }^{1}$, Hye Gyung Rheu, M.D., Ph.D. ${ }^{2}$ \\ Departments of ${ }^{1}$ Surgery and ${ }^{2}$ Internal Medicine, Suh-Gwang General Hospital, Gwangju, Korea
}

Two or more procedures maybe combined into a single surgical event using an abdominal laparoscopic surgery technique. Synchronous operations can provide patients with the advantage of a single hospital stay, single anesthetic exposure and single recovery period. Cholecystectomy and totally extraperitoneal (TEP) herniorrhaphy should be performed in both extremities and in different spaces of the abdomen. As described in this report, laparoendoscopic single site surgery (LESS), synchronous cholecystectomy and TEP herniorrhaphy were successfully performed using an umbilical incision in a single surgical event.

Key words: Cholecystectomy, TEP herniorrhaphy, Hernia, LESS

\section{INTRODUCTION}

Minimally invasive surgeons have begun to use single-incision access to perform wide range of surgical operations and have been used successfully to obtain good results comparing to their novel procedures. ${ }^{1}$ With advancement of laparoendoscopic single-site surgery (LESS) a number of procedures of abdomen can be performed in a single surgery. ${ }^{2}$ For the synchronously diagnosed gallstones and inguinal hernia, combined operation of cholecystectomy and totally extraperitoneal (TEP) herniorrhaphy would be the most minimally invasive treatment in the era of LESS. But anatomically, the gallbladder and inguinal hernia is located in the both extremities in the abdomen. And these procedures are performed in the different space. Cholecystectomy is done in the intraperitoneal space of upper abdomen, and TEP herniorrhaphy in the preperitoneal space of lower abdomen.

We report a successfully performed Synchronous cholecystectomy and TEP herniorrhaphy using an umbilical incision in a single surgery.

\section{CASE REPORT}

A 66-year-old man in whom multiple gallstones and right indirect inguinal hernia has been diagnosed was referred to our

Received June 12, 2012, Accepted July 2, 2012

* Corresponding author: Hae-Hyeon Suh

Department of Surgery, Suh-Gwang General Hospital, 250-24,

Geumho-dong, Seo-gu, Gwangju 502-803, Korea

Tel : +82-62-600-8123, Fax : +82-62-600-8199

E-mail : suhhh2000@ hanmail.net

http://dx.doi.org/10.7602/jmis.2012.15.3.79 hospital for further evaluation and treatment. He was admitted with a complaint of right inguinal protruding mass with vague right lower abdominal pain for 2 years and intermittent discomfort of right upper abdomen for 5 years. His medical history was hypertension medication for 5 years. A physical examination revealed spontaneously reducible bulging mass extending from inguinal canal to the proximal scrotum. No specific findings were noted in the upper abdomen. An abdominal computed tomography demonstrated non-complicated right indirect inguinal hernia and multiple gallstones with chronic cholecystitis (Fig. 1, 2).

We underwent synchronous cholecystectomy and totally extraperitoneal (TEP) herniorrhaphy using an umbilical incision under general anesthesia with endotracheal intubation in supine position. The procedures were performed with a single port device (Gloveport ${ }^{\mathrm{TM}}$; 4-channel, Sejong, Seoul, Korea) via a 2.5 $\mathrm{cm}$ transverse umbilical incision using a straight $10 \mathrm{~mm} 30$ degree laparoscope and articulating instruments. After the skin incision, the subcutaneous fat and right side of the anterior rectus sheath were dissected bluntly. Retracting the rectus muscle anterolaterally, right index finger was inserted into the preperitoneal space of right lower abdomen for blunt dissection to prepare the space to insert a single port device. The port was inserted into the preperitoneal space. Carbon dioxide gas was insufflated into the preperitoneal space with $12 \mathrm{mmHg}$ pressure. Using blunt and sharp dissection, the space was created and the anatomical configuration was confirmed (Fig. 3). The hernia sac was identified, isolated, cut and ligated. A peritoneal opening made during the preperitoneal dissection was closed using a loop ligation. A $12 \times 15 \mathrm{~cm}$ sized polypropylene mesh was secured with a tacking device. After hemostasis was confirmed, the air was removed under direct vision trying 


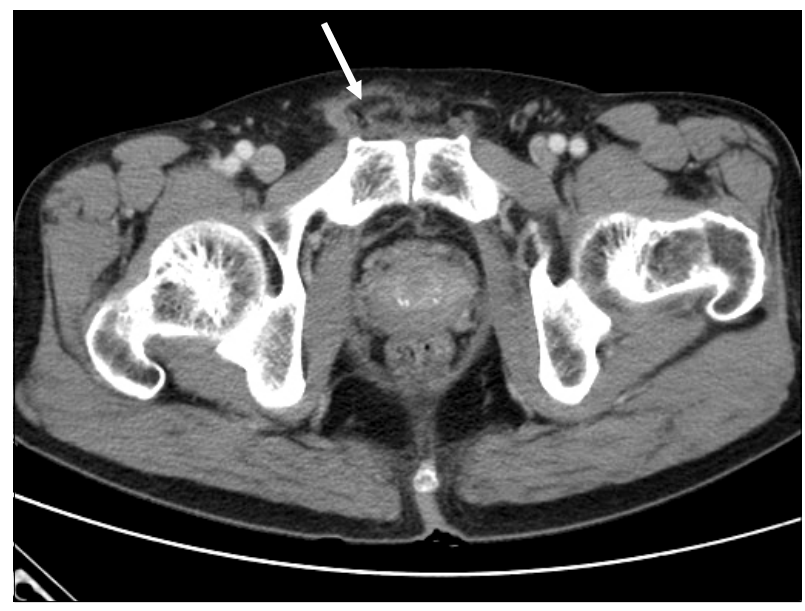

Fig. 1. A pelvic computed tomography (CT) demonstrates right inguinal canal filled with vasculoadipose tissues (arrow) indicating indirect inguinal hernia.

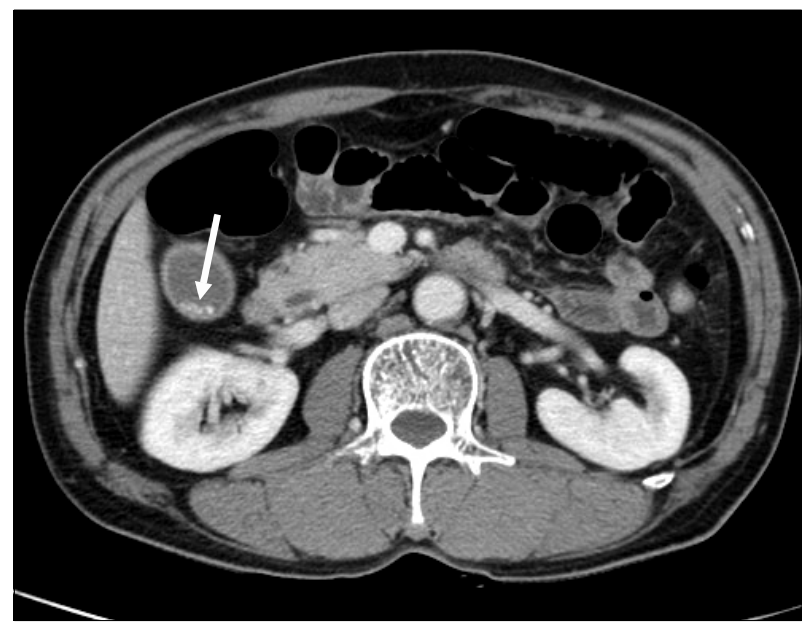

Fig. 2. An abdominal computed tomography (CT) presents multiple gallstones (arrow) with chronic cholecystitis.

to keep proper position of the mesh.

Intraperitoneal approach for the cholecystectomy was performed after the port was removed. The posterior rectus sheath and the peritoneum were opened bluntly and the port used for the previous procedure was inserted into the peritoneal cavity. The gallbladder was adhered loosely by the omentum and revealed no hyperemia or edema. In the anterolateral side of the chest a thread of 5-0 silk and straight needle was passed from the skin into the peritoneal cavity through the intercostal space and pierced the gallbladder. The needle was retrieved through the same space and the silk was used for retracting the gallbladder. Careful dissection using ultrasonic scissors revealed critical structures of the Calot's triangle (Fig. 4). The cystic duct was double clipped and cut. The gallbladder dissected

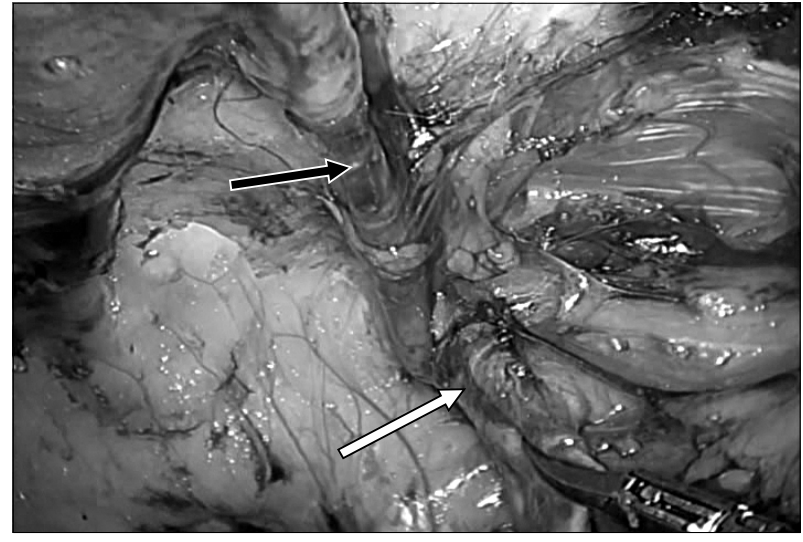

Fig. 3. Totally extraperitoneal (TEP) dissection revealed hernia sac (white arrow) and epigastric vessels (black arrow).

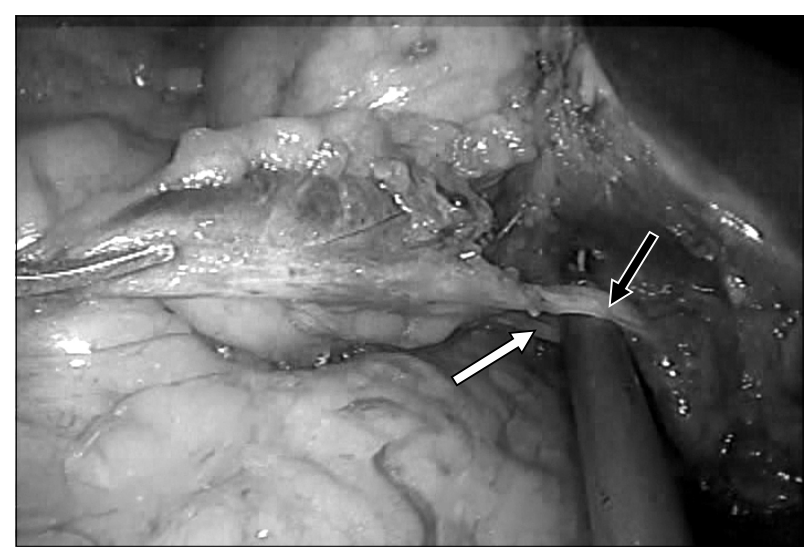

Fig. 4. Critical view of structures at the triangle of Calot revealed cystic duct (black arrow) and cystic artery (white arrow).

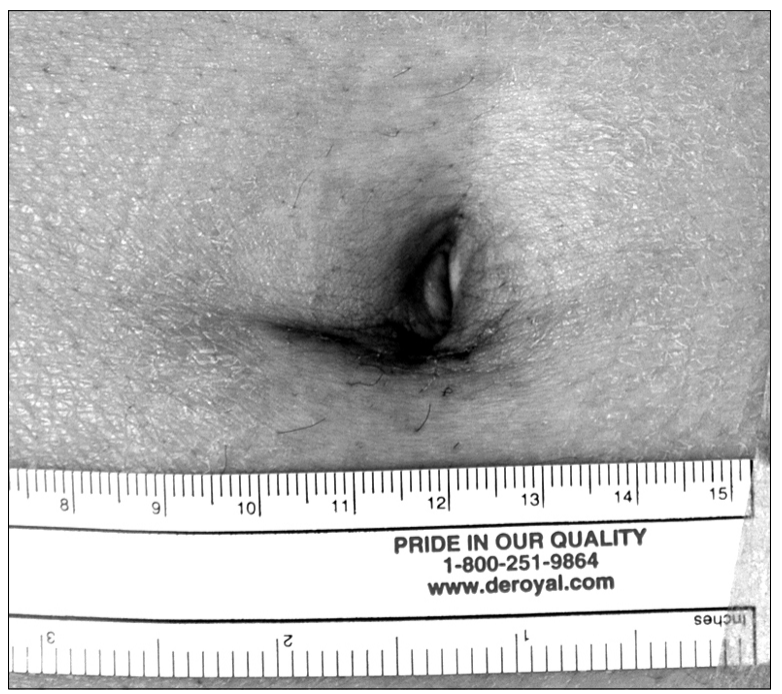

Fig. 5. Good aesthetic results of umbilical wound, checked 2 weeks after LESS synchronous cholecystectomy and TEP herniorrhaphy. 
from the liver was retrieved using a specimen bag. There was no significant bleeding or bile leakage in the operation field. The wound was closed layer by layer without leaving a drain. Total duration of the surgical operation was 98.9 minutes. Duration from the skin incision to the end of TEP herniorrhaphy was 64.6 minutes. The operation time of the cholecystectomy until the wound closure was 34.3 minutes. There was no significant postoperative complication. Postoperative hospital stay was 4 days. Time to resume work was 5 days after surgery. Two weeks postoperative scar showed $2.5 \mathrm{~cm}$ in length with favorable cosmetic result (Fig. 5).

\section{DISCUSSION}

Minimally invasive surgery intend to achieve a scarless approach, improved cosmesis, better pain profile and better quality of life. ${ }^{3,4}$ Patients seem to favor scarless surgery over standard multiport laparoscopy. ${ }^{5}$ Natural orifice transluminal endoscopic surgery (NOTES) and LESS surgery are significant new development in the continuing evolution of minimally invasive therapies. LESS surgery can be an alternative to conventional laparoscopic (CL) surgery associated with less traumatic to patient enhancing early recovery, better cosmesis, body image, quality of life and improved postoperative pain profile without interfering economic aspect. ${ }^{6}$ Transumbilical LESS surgery raises the dream of near-complete cosmesis. The laparoscopic surgery of abdomen provides an excellent view of the entire abdomen which inspires surgeons to apply combining two or more procedures in a single surgery. Synchronous operation can provide patients with the advantage of single hospital stay and single anesthetic exposure resulting in convenience and cost effectiveness although combining procedures cause longer operation time, longer anesthesia, and risk of increased blood loss. ${ }^{7}$

For LESS synchronous operations of cholecystectomy and TEP herniorrhaphy, only one umbilical incision was required although they were located in the both extremities of abdomen. If conventional laparoscopic techniques were adopted, it would require more than six port incisions, three or four for upper abdomen and three for lower abdomen.

Laparoscopic cholecystectomy using three or four ports is widely accepted as the standard operation for benign gallbladder disease. Transumbilical LESS cholecystectomy was first described in the 1990s, but has not been accepted widely due to technical difficulties. Recently, with development of techniques and technologies, reports of LESS cholecystectomy have demonstrated its feasibility. ${ }^{8}$ The critical view of safety was achieved with technical improvement and instrumental development. LESS cholecystectomy can be an alternative to conventional laparoscopic (CL) cholecystectomy associated with better cosmesis, body image, quality of life and improved postoperative pain profile. $^{6}$

Open, tension free, mesh repair has long been the gold standard of inguinal herniorrhaphy. In contrast to other intraabdominal procedures, surgeons have been resistant to the adoption of laparoscopic inguinal herniorrhaphy ( $\mathrm{LIH})$. The main reasons of criticism were technical difficulties, cost and a long learning curve. ${ }^{8}$ Although transabdominal preperitoneal (TAPP) technique requires intraabdominal procedures leading to the possibilities of injury to the intraperitoneal contents, TEP herniorrhaphy has no need to enter the peritoneal cavity. ${ }^{9}$ Although TEP herniorrhaphy is superior to tension free open herniorrhaphy in terms of postoperative pain and rehabilitation, learning the procedure is difficult. The main reasons of the extremely steep learning curve are unfamiliar anatomical structures of the preperitoneal space and technical difficulties, mainly loss of triangulation. Because the major drawback of LESS surgery is loss of triangulation, the surgeon who has already skilled in multiport LIH can transit to LESS surgery without increasing operative time or morbidity in TEP herniorrhapny. ${ }^{10}$

We underwent TEP herniorrhaphy before cholecystetomy. The reasons of the sequence were (1) the preperitoneal space locates in front of the peritoneal space anatomically and the intact peritoneum is better to introduce the port to the prepreitoneal space; (2) if pneumoperitoneum is caused by the torn peritoneum during TEP procedure, it would be harmless to do next procedure, rather beneficial to making a peumoperitoneum for cholecystectomy; (3) if cholecystectomy is performed first, intraperitoneal air remained after the procedure could interfere TEP procedure.

In conclusion, we underwent synchronous cholecystectomy and TEP herniorrhaphy using an umbilical incision. All procedures were performed with a single port device via a $2.5-\mathrm{cm}$ umbilical incision using a rigid 30 degree $10-\mathrm{mm}$ laparoscope and articulating instrument.

\section{REFERENCES}

1) Gill IS, Advincula AP, Aron M, et al. Consensus statement of the consortium for laparoendoscopic single-site surgery. Surg Endosc 2010;24:762-768.

2) Wadhwa A, Chowbey PK, Sharma A, et al. Combined procedures in laparoscopic surgery. Surg Laparosc Percutan Tech 2003;13:382-386. 
3) Suh HH. A minimally invasive technique of appendectomy using a minimal skin incision and laparoscopic instruments. Surg Laparos Endos 1998;8:149-152.

4) Pelosi MA, Pelosi MA 3rd. Laparoscopic appendectomy using a single umbilical puncture (minilaparoscopy). J Reprod Med 1992;37:588-594.

5) Bucher P, Pugin F, Ostermann S, et al. Population perception of syrgical safety and body image trauma: a plea for scarless surgery? Surg Endosc 2011;25:408-415.

6) Bucher P, Pugin F, Buchs NC, et al. Randomized clinical trial of laparoendoscopic single-site versus conventional laparoscopic cholecystectomy. Br J Surg 2011;98:1695-1702.
7) Wadhwa A, Chowbey PK, Sharma A, et al. Combined procedures in laparoscopic surgery. Surg Laparosc Endosc Percutan Tech 2003; 13:382-386.

8) Edwards CC 2nd, Bailey RW. Laparoscopic hernia repair: the learning curve. Surg Laparosc Endosc Percutan Tech 2000;10: 149-153.

9) Bringman S, Ramel S, Heikkinen TJ, et al. Tension-free inguinal hernia repair: TEP versus mesh-plug versus Lichtenstein: a prospective randomized controlled trial. Ann Surg 2003;237: 142-147.

10) Sherwinter DA. Transitioning to single-incision laparoscopic inguinal herniorrhaphy. JSLS 2010;14:353-357. 